\title{
Vitamin D and Peripheral Vascular Resistance: Are They Really Related?
}

\author{
K. Bhaskar ${ }^{1} \quad$ K. Swaroopa ${ }^{2}$ \\ ${ }^{1}$ Department of Pulmonary Medicine, Nizams Institute of Medical \\ Sciences, Hyderabad, India \\ ${ }^{2}$ Department of General Medicine, Nizams Institute of Medical \\ Sciences, Hyderabad, India \\ Ind J Car Dis Wom 2019;4:121
}

Vitamin D is one of the fat-soluble vitamins that has been a focus of increasing attention in recent times due to its presence in all tissues. Although traditionally described in bone and calcium metabolism, research has shown its active role in various other physiological processes of cardiovascular, respiratory, immune systems, and metabolism of lipids and sugars. Vitamin D receptors are known to be present in all parts of vascular sheath like endothelium, smooth muscle cells suggesting its active role in maintaining homeostasis.

Vitamin D deficiency has been shown in association with various disorders like hypertension, diabetes, asthma, cardiovascular, peripheral vascular, cancer, and autoimmunity. Vitamin D deficiency is known to be associated with vasoconstriction leading to increased stiffness and peripheral vascular disease.

Vascular stiffness comprises various physical parameters like compliance, distensibility, and elasticity. Clinically these are measured with pulse wave velocity (PWV) and augmentation pressure (AG)/augmentation Index (AI). Vitamin D levels were inversely associated with PWV and AI but supplementation has not yielded consistent positive results.

In a study by Murat Sunbul supplementation of vitamin D has improved PWV and AI in patients with deficiency. ${ }^{1}$ In another study done by Chitalia et al, vitamin D showed positive effect on endothelial function but no such effect on arterial stiffness in chronic kidney disease patients. ${ }^{2}$ Tsuru et al studied the effect of age and gender on AI and AG with findings of continued increase in AG with age, whereas AI varied with gender, height, and type of vessel leading to final conclusion of AG as a suitable parameter of arterial stiffness than $\mathrm{Al}^{3}{ }^{3}$

Chua et al, in a review, opined that vitamin D deficiency is an independent risk factor for peripheral vascular disease that can be corrected easily. ${ }^{4}$
Vitamin D deficiency acts as an epigenetic factor tilting the balance toward vasoconstriction, thereby predisposing the development of essential hypertension.5

In this issue of Indian Journal of Cardiovascular Diseases in Women, authors tried to analyze the relationship between vitamin D insufficiency and deficiency with peripheral vascular resistance (PVR). ${ }^{6}$ They have assessed PVR, cardiac output, cardiac index, systolic blood pressure, PWV, AG, and AI. High PVR and systolic blood pressure were observed in majority of these patients without any gender difference. There was no statistical significance in AG and AI in both genders. This study could not find any gender variations with respect to above parameters. Small size is the main drawback and further prospective studies with large sample are needed to substantiate these findings. This study can be considered for publication as there is lot of growing interest in this topic.

\section{Conflicts of Interest}

None declared.

\section{References}

1 Sünbül M, Çinçin A, Bozbay M, et al. Arterial stiffness parameters associated with vitamin D deficiency and supplementation in patients with normal cardiac functions. Turk Kardiyol Dern Ars 2016;44(4):281-288

2 Chitalia N, Recio-Mayoral A, Kaski JC, Banerjee D. Vitamin D deficiency and endothelial dysfunction in non-dialysis chronic kidney disease patients. Atherosclerosis 2012;220(1):265-268

3 Tsuru T, Adachi H, Enomoto M, et al. Augmentation index (AI) in a dose-response relationship with smoking habits in males: The Tanushimaru study. Medicine (Baltimore) 2016;95(51):e5368

4 Chua GT, Chan YC, Cheng SW. Vitamin D status and peripheral arterial disease: evidence so far. Vasc Health Risk Manag 2011;7:671-675

5 Chen S, Sun Y, Agrawal DK. Vitamin D deficiency and essential hypertension. J Am Soc Hypertens 2015;9(11):885-901

6 article going to publish in same issue [6]

\section{Address for correspondence} K. Swaroopa,MBBS, MD, Department of General Medicine, Nizams Institute of Medical Sciences, Hyderabad, 500082, India (e-mail: swaroopareddymd@gmail.com).
DOI https://doi.org/ 10.1055/s-0039-3400198
C2019 Women in Cardiology and Related Sciences
License terms

$(\circledast)(1) \Theta \circledast$ 